\title{
WEAK GARDENS OF EDEN FOR 1-DIMENSIONAL TESSELLATION AUTOMATA
}

\author{
MICHAEL D. TAYLOR \\ Mathematics Department \\ University of Central Florida \\ orlando, Florida \\ (Received July 16, 1984)
}

ABSTRACT: If $T$ is the parallel map associated with a 1 -dimensional tessellation automaton, then we say a configuration $f$ is a weak Garden of Eden for $T$ if $f$ has no pre-image under $T$ other than a shift of itself. Let $W G(T)=$ the set of weak Gardens of Eden for $T$ and $G(T)=$ the set of Gardens of Eden (i.e., the set of configurations not in the range of T). Typically members of $W G(T)-G(T)$ satisfy an equation of the form $T f=S^{m_{f}}$ where $S^{m}$ is the shift defined by $\left(S^{m} f\right)(j)=f(j+m)$. Subject to a mild restriction on $m$, the equation $\mathrm{Tf}=\mathrm{S}^{\mathrm{m}} \mathrm{f}$ always has a solution $\mathrm{f}$, and all such solutions are periodic. We present a few other properties of weak Gardens of Eden and a characterization of WG(T) for a class of parallel maps we call $(0,1)$-characteristic transformations in the case where there are at least three cell states.

KEY WORDS AND PIRASES: Celzular automata, tessellation automata, Cardens of Edi=ri, firalizel maps.

1380 MATHEMATICS SUB.'ECT CLASSIFICATION CODE. 68D::

1. INTRODUCI'IN.

A Garden of Eden configuration for a tessellation automaton is one which has no predecessor under application of the local transition function; it must be a "given" configuration, hence the colorful name. By a weak Garden of Eden we mean a configuration which has no predecessor other than perhaps some shift of itself. To simplify matters, we will consider only 1-dimensional tessellation automata. We illustrate here a technique which can often be used to manufacture periodic weak Gardens of Eden for parallel maps. We also present a few simple results: For example, one-to-one parallel maps always have weak Gardens of Eden. Finally we consider $(0,1)$-characteristic parallel maps. A $(0,1)-$ characteristic parallel map is defined in the following way: Let $t$ be its local transition function. There must be a given word a such that for all words b having the same length as a we have

$$
t(b)=\left\{\begin{array}{l}
l \text { if } b=a \\
0 \text { otherwise }
\end{array}\right.
$$

(We assume, of course, that $0,1, a$, and b are made up of symbols from our set of states for our tessellation automaton.) This set of characteristic parallel maps is ubiquitous in the sense that all other parallel maps can be constructed from combinations of them. We give a characterization of the Gardens of Eden of the $(0,1)$-characteristic parallel 
maps and then a characterization of those weak Gardens of Eden which are not Garden of Eden for the same class of maps under the additional assumption that the cells of the tessellation automata have at least three possible states.

2. PRELIMINARIES

Let $A$ be a fixed, finite set which we think of as an alphabet or set of states or "colors". We always assume 0 and 1 are members of A. By a (1-dimensional) configuration we mean a function $\mathrm{f}: \mathrm{Z} \rightarrow \mathrm{A}$ where $\mathrm{Z}$ is the set of integers. Let $\mathrm{C}$ be the set of all configurations. By a string we mean a function $f: J \rightarrow A$ where $J$ is an interval in $Z$. If $J=[j+1, j+m]=\{j+1, j+2, \ldots, j+m\}$, then we say the string has length $\mathrm{m}$; we do not in general demand that strings have finite length. If $g$ is a configuration, then the restriction of $g$ to an interval in $Z$ is called a string in $g$.

Note that we use the term string in a way which is not quite standard. Usually "string" is taken to be synonomous with "word", and by a word over A one means a finite sequence of elements of $A$. The differences between the two concepts are that strings may have infinite lengths and that one specifies where a string is in $z$ (i.e., one gives an interval in $\mathrm{Z}$ as its domain). We say more about this in section 4.

By a local map or local transition function we mean a function of the form $t: A^{n} \rightarrow A$ where $A^{n}$ is the $n$-fold cartesian product of $A$ with itself. We can then define $t: C^{n} \rightarrow C$ (note this is the same symbol $t$ as in the last sentence but a different function) in pointwise fashion:

$$
\left(t\left(f_{1}, f_{2}, \ldots, f_{n}\right)\right)(j)=t\left(f_{1}(j), f_{2}(j), \ldots, f_{n}(j)\right)
$$

where $f_{1}, f_{2}, \ldots, f_{n} \in C$ and $j \in Z$. If Map $(C, C)=$ the set of functions from $C$ to $C$, then we can define yet a third $t$, this time

$$
\mathrm{t}: \operatorname{Map}(\mathrm{C}, \mathrm{C}) \rightarrow \operatorname{Map}(\mathrm{C}, \mathrm{C})
$$

by the standard pointwise extension technique:

$$
\left(t\left(T_{1}, T_{2}, \ldots T_{n}\right)\right)(f)=t\left(T_{1}(f), T_{2}(f), \ldots, T_{n}(f)\right)
$$

where $T_{1}, T_{2}, \ldots T_{n} r \operatorname{Map}(C, C)$ and $f \in C$.

EXAMPLE 1 . Let $A=\{0,1, \ldots, p-1\}$ and suppose we endow $A$ with the algebraic structure of $Z /(p)$, the integers modulo $p$. Define $t: A^{2} \rightarrow A$ by $t(x, y)=x+y$. Then for $\mathrm{f}_{1}, \mathrm{f}_{2} \in \mathrm{C}$ and $\mathrm{T}_{1}, \mathrm{~T}_{2} \in \operatorname{Map}(\mathrm{C}, \mathrm{C})$ we would write $t\left(\mathrm{f}_{1}, \mathrm{f}_{2}\right)=\mathrm{f}_{1}+\mathrm{f}_{2}$ and $t\left(T_{1}, T_{2}\right)=T_{1}+T_{2}$.

By a shift (or translation) $S^{p}$, where $p \in Z$, we mean the function $S^{p}: C \rightarrow C$ defined by $\left(S^{p}(f)\right)(j)=f(j+p)$ where $f \in C$ and $j \in Z$. Now let $w=[j+1, j+n]$, a finite interval in $\mathrm{Z}$. If $\mathrm{t}: \mathrm{A}^{\mathrm{n}} \rightarrow \mathrm{A}$ is a local map, then the parallel map (or global map or window-transformation or W-transformation) $T$ with window $W$ defined by $t$ is the function $T: C \rightarrow C$ defined by $T=t\left(s^{j+1}, s^{j+2}, \ldots, s^{j+n}\right)$.

EXMPLE 2. Using $t$ and A from Example 1 and setting $W=\{2,3\}$, we obtain $T=t\left(S^{2}, S^{3}\right)=S^{2}+S^{3}$. Then for $f \in C$ and $i \epsilon Z$ we have $(T f)(i)=f(i+2)+f(i+3)$.

NOTE. In this example we wrote Tf instead of $T(f)$, and we will continue this convention throughout this paper.

For $T$ a parallel map we say a configuration $f$ is a Garden of Eden provided it is not in the range of $T$. Information about Gardens of Eden can be found in [1], [2], $[3],[4],[5]$, and [ 6$]$. We say $\mathrm{f}$ is a weak Garden of Eden of $\mathrm{T}$ provided that whenever 
$\mathrm{Tg}=\mathrm{f}$, then $\mathrm{g}$ must be of the form $\mathrm{S}^{\mathrm{k}} \mathrm{f}$ for some $k$. Let $\mathrm{G}(\mathrm{T})=$ the set of Gardens of Eden of $T$ and $W G(T)=$ the set of weak Gardens of Eden. We see that $G(T) \subset W G(T)$.

\section{ELFMFNTARY PROPERTIES OF WEAK GARDENS OF EDEN}

PROPOSITION 1. If $\mathrm{f}$ is a weak Garden of Eden, but not a Garden of Eden for the parallel maps $\mathrm{T}$, the $\mathrm{Tf}=\mathrm{S}^{\chi} \mathrm{f}$ for some integer $\ell$.

PROOF. Since $f$ must be in the range of $T$, we can find $g$ such that $T g=f$. But $g=s^{k} f$ for some integer $k$. As parallel maps commute with shifts, we have $\mathrm{Tf}^{\mathrm{k}}=\mathrm{s}^{-\mathrm{k}} \mathrm{f}$. Suppose the parallel map $T$ is defined by $T=t\left(S^{j+1}, \ldots, S^{j+n}\right)$ where $t$ is a local map. We say $t$ is permutive in the ith variable (or hyperactive in the ith variable) provided that whenever we chose $a_{1}, a_{2}, \ldots, a_{n}, b_{1}, b_{2}, \ldots, b_{n}$ from A with the properties that $a_{i} \neq b_{i}$ but $a_{k}=b_{k}$ for $k \neq i$, then $t\left(a_{1}, a_{2} \ldots, a_{n}\right) \neq t\left(b_{1}, b_{2}, \ldots b_{n}\right)$. We say $\mathrm{T}$ is permutive (or hyperactive) in the ith variable if and only if $t$ is.

If $J$ is an interval in $Z$ and $r$ an integer and $f$ and $g$ are functions from $J$ and $J+r$ respectively into $A$, we say $g$ is a copy of $f$ provided that $g(j+r)=f(j)$ for every $j \subset J . \quad($ Note: $J+r=\{j+r: j \subset J\}$.

PROPOSITION 2. Let $T$ be a parallel map with window $W$. For every integer $l d W$, the equation Tf $=\mathrm{S}^{\ell} \mathrm{f}$ has a solution $\mathrm{f}$. Further, every solution is periodic.

PROOF. We may consider $A$ to be $\{0,1,2, \ldots, p-1\}$ and endow it with the algebraic structure of $Z /(p)$. As pointed out before, this structure maybe lifted in a pointwise fashion onto the configurations and then onto the set of maps of $C$ into $C$. Then the equation $\mathrm{Tf}=\mathrm{S}^{2} \mathrm{f}$ maybe rewritten $\left(\mathrm{T}-\mathrm{S}^{l}\right.$ ) $\mathrm{f}=0$ (the constant 0 configuration). Since ' W, it follows that $T-S^{\ell}$ is permutive in its first or last variable. The fact that there is a solution follows from Theorem 6.6 of $[7]$ and the periodicity of $f$ from Theorem 9.1 of [7] and the periodicity of the constant 0 configuration.

EXAMPLE 3. We illustrate how one may sometimes manufacture weak Gardens of Eden by using the ideas in Propositions 1 and 2 . Let $A=\{0,1\}$ (which we identify with $Z /(2)$ ) and set $\mathrm{T}=\mathrm{S}^{1} \cdot\left(1+\mathrm{S}^{0}\right)$ (note that we are using both multiplication and addition mod 2 ). Our window is $W=[0,1]$. Let us seek a weak Garden of Eden satisfying $T f=S^{3} f$. Such an $f$ must satisfy $f(j+3)=f(j+1) \cdot(1+f(j))$ for every integer $j$. We start with a "seed" string, for example 001 , and use the recurrence relation on $f$ to extend it indefinitely to the right. The result is $00101010101 \ldots$ (The length of the "seed" one starts with is readily determined from the length of $W$ and the distance of $\ell$ from $W$; the choice of the "seed" string is a matter of experimentation.) We see from this that we might try the configuration $f$ given by ... $01010101 \ldots$ It is easily checked that the only pre-image of $f$ under $T$ is a shift of $f$ one unit to the right or left.

EXAMPLE 4. For any shift $s^{k}$, we have

$$
G\left(S^{k}\right)=p \text { and } W G\left(S^{k}\right)=C \text {. }
$$

EXAMPLE 5. If $t: A+A$ is a permutation of $A$ and $T=t\left(S^{k}\right)$, then every $f$ satisfying Tf $=S^{\vartheta} f$ for $; \neq k$ is a weak Garden of Eden. Such $f^{\prime}$ 's are easily manufactured by starting with a "seed" string and appealing to $t(f(j+k))=f(j+l)$.

EXAMPLE 6 . We give an example of a weak Garden of Eden which is not a Garden of Eden and is not periodic. Let $A=\{0,1\}$ and $T=\left(1+S^{0}\right) \cdot\left(1+S^{1}\right) \cdot S^{2} \cdot\left(1+S^{3}\right)$. l.et $f$ be a configuration containing an infinite number of 1 's such that any two successive 1 's are separated from one another by either two $0^{\prime}$ 's or three 0 's and $f$ is not periodic. 
A portion of $f$ might well look like this

$$
\ldots 1001000100100100100010001001 \ldots
$$

Because $(\Gamma f)(j)=1$ only when $f(j)=f(j+l)=f(j+3)=0$ and $f(j+2)=1$, it is easily checked that the only pre-image of $f$ is $s^{-2} f$. That means that $\mathrm{Tf}=\mathrm{s}^{2} \mathrm{f}$ so that $2=r, W=[0,3]$ and Proposition 2 does not apply.

PROPOSITION 3. Every weak Garden of Eden has at most a single pre-image.

PROOF: Let $f \in W G(T)-G(T)$ and suppose $T g=T h=f$. There must exist integers $\ell$ and $m$ such that $g=S^{\chi} f$ and $h=s^{m} f$. Then $T\left(S^{?} f\right)=T\left(S^{m} f\right)=f$ so that $s^{-l} f=T(f)=S^{-m} f$. Hence $s^{m-x} f=f$. Therefore $h=s^{m} f=\left(s^{l} \circ s^{m-l}\right) f=s^{l} f=g$.

EXAMPLE 7. Let $A=\{0,1, \ldots, p-1\}$ and treat it as $Z /(p)$. Set $T=S^{1}-S^{0}$. To say that $\mathrm{Tg}=\mathrm{f}$ is to say that $g(j+1)-g(j)=f(j)$ for every integer $j$. One can see from this that $T$ is onto and that every $f$ has exactly $|A|$ pre-images where $|A|=$ the cardinality of A. Then by proposition $3, W G(T)=+$.

We note also that information about parallel maps which are h-to-one can be found in [7], [8], and [9] and that if $h \geq 2$, then $W G(T)-G(T)=\phi$.

PROPOSITION 4. Every one-to-one parallel map has a weak Garden of Eden which is not a Garden of Eden.

PROOF : Let $T$ be a one-to-one parallel map, and let $\ell$ be an integer not in $T^{\prime} s$ window. Then $\mathrm{Tf}=\mathrm{S}^{2} \mathrm{f}$ has a solution $\mathrm{f}$. This means $\mathrm{T}\left(\mathrm{S}^{-\ell} \mathrm{f}\right)=\mathrm{f}$. But $\mathrm{f}$ has a unique pre-image, so it must be the desired configuration.

PROPOSITION 5. If $T$ and $Q$ are parallel maps such that $Q$ is one-to-one and we define the parallel map $R$ by $R=Q^{-1}$ o $T$ o $Q$, then $Q(G(R))=G(T)$ and $Q(W G(R)-G(R))=W G(T)-G(T)$.

PROOF. Note that by [10] we known $Q^{-1}$ must be a parallel map, and hence so is $R$. It is straightforward to show $Q(G(R))=G(T)$, so we consider only the second half of the conclusion. Let $f \circ W G(T)-G(T)$. We need only show that $Q^{-1} f \in W G(R)-G(R)$. Clearly $Q^{-1} \mathrm{f} \notin \mathrm{G}(\mathrm{R})$ since $\mathrm{f} \notin \mathrm{G}(\mathrm{T})$. Suppose $\mathrm{g}$ is a configuration such that $\mathrm{Rg}_{\mathrm{g}}=Q^{-1} \mathrm{f}$. Then $T(Q g)=f$ and thus $Q g=S^{\ell} f$ for some integer $\ell$. Hence $g=S^{k}\left(Q^{-1} f\right)$. Therefore

$$
W G(T)-G(T) \subset Q(W G(R)-G(R))
$$

Lin. $t$ T () $\circ \mathrm{K} O \mathrm{Q}^{-1}$, containment in the other direction also holds. []

PROPOSITION $\mathrm{h}$. For any parallel maps $\mathrm{T}_{1}, \mathrm{~T}_{2}, \ldots, \mathrm{T}_{\mathrm{n}}$ we have $W G\left(T_{1}\right) \cap \ldots \cap W G\left(T_{n}\right) \subset W G\left(T_{1} \circ \ldots O_{T_{n}}\right)$.

PROOF. Let $f \in W G\left(T_{1}\right) \cap \ldots \cap W G\left(T_{n}\right)$ and suppose $\left(T_{1} \circ \ldots \circ T_{n}\right) g=f$. There is an integer $i_{1}$ such that $\left(\mathrm{T}_{2} \circ \ldots \circ \mathrm{O}\right) \mathrm{g}=\mathrm{S}^{\ell} 1 \mathrm{f}$ or, equivalently, $\left(\mathrm{T}_{2} \circ \ldots \circ \mathrm{T}_{\mathrm{n}}\right)\left(\mathrm{S}^{-\ell} 1 \mathrm{~g}\right)=\mathrm{f}$. Clearly we can continue this process to produce an integer $\ell$ such that $g=s^{\ell} f$.

4. CHARACTERISTIC PARALLEL MAPS.

Let $a$ and $b$ be distinct elements of $A$ and $\left(a_{1}, a_{2}, \ldots, a_{n}\right)$ a given ordered $n$-tuple in $A^{n}$. We call $t: A^{n} \rightarrow A$ the $(\underline{a, b})$-characteristic local map for $\left(a_{1}, a_{2}, \ldots a_{n}\right)$ provided

$$
t\left(b_{1}, b_{2}, \ldots b_{n}\right)=\left\{\begin{array}{l}
b \text { if }\left(b_{1}, b_{2}, \ldots, b_{n}\right)=\left(a_{1}, a_{2}, \ldots, a_{n}\right) \\
\text { and } \\
\text { a otherwise. }
\end{array}\right.
$$

We then call $\mathrm{T}$ an $(\mathrm{a}, \mathrm{b})$-characteristic parallel map for $\left(\mathrm{a}_{1}, \mathrm{a}_{2}, \ldots, \mathrm{a}_{\mathrm{n}}\right)$ provided it is of the form $T=t\left(S^{j+1}, S^{j+2}, \ldots, s^{j+n}\right)$ for some integer $j$. We will consider only $(0,1)-$ characteristic parallel maps and for brevity will refer to them as characteristic maps. 
We min. 'hat all parallel maps can be built up from characteristic maps. Let $A=\{0,1, \ldots, p-1\}$ and 1 let us treat it as the integers $\bmod p$. Let $s_{1}, s_{2}, \ldots, s_{N}$ be all the ordered n-tuples in $A^{n}$, and for each $i$ let $t_{i}$ be the characteristic local map for $s_{i}$. If $t: A^{n} \rightarrow A$ is a local map and $c_{i}=t\left(s_{i}\right)$ for every $i$, then $t=c_{1} t_{1}+\ldots+c_{N} t_{N}$. Take a window $w=[j+l, j+n]$ in the integers and for each 1 let $T_{i}$ be the characteristic map $t_{i}\left(S^{j+1}, S^{j+2}, \ldots S^{j+n}\right)$. If $T$ is the parallel map defined by $t$ and having window $w$, then we can write $T=c_{1} T_{1}+\ldots+c_{N} T_{N}$.

In this section we give characterizations of $G(T)$ and $W G(T)-G(T)$ for $T$ a characteristic map. In the case of WG(T) - G(T) we assume A contains at least three elements.

Before beginning we need some terminology.

Recall that by a word over $\underline{A}$ we mean an ordered sequence $\left(a_{1}, a_{2}, \ldots a_{m}\right)$ of elements of $A$, but we use the symbolism $a_{1} a_{2} \cdots a_{m}$ for it. If $a=a_{1} a_{2} \ldots a_{m}$ and $b_{=} b_{1} b_{2} \ldots b_{n}$, two words over $A$, then we can concatenate them to produce a single word $a b=a_{1} a_{2} \cdots a_{m} b_{1} b_{2} \cdots b_{n}$. We assume the existence of an empty word $z$ such that $a z=z a=a$ for every word $a$. If $a=a_{1} a_{2} \cdots a_{m}$ where the $a_{i}$ 's are elements of $A$, then the length of $a$ is $|a|=m$. If $a, b$, and $c$ are words such that $a=b c$, we say $b$ is $a \underline{\text { left }} \underline{\text { factor of } a \text {; }}$ if $c$ is not the empty word, we say $b$ is proper left factor of a. Right factors and proper right factors are defined in a similar fashion. If $c \in A$, then $c^{1}=c$ and $c^{n+1}=c^{n} c$ where $n=1,2, \ldots$

clearly strings of finite length are almost the same thing as words. If we have a string $f: J \rightarrow$ A where $J=[j+1, j+m]$ and $f(j+i)=a_{i}$ for $i=1,2, \ldots, m$, then we say $f$ is a copy of the word $a_{1} a_{2}, \ldots a_{m}$, and we even permit ourselves to write (abusing notation slightly) $f=a_{1} a_{2} \ldots a_{m}$. If $h$ is a configuration and $J$ an interval in $z$, then we say $\underline{h}$ has a copy of the word $\underline{\text { a }} \underline{\text { at }} \mathrm{J}$ provided $\mathrm{h} / \mathrm{J}$, considered as a string, is a copy .. a.

Il is also useful to define what we mean by successive occurrences of symbuls and words in a configuration. Let $f$ be a configuration. For an element $b$ of $A$, we say that distinct integers $i$ and $j$ mark successive occurrences of $b$ in $f$ provided $f(i)=f(j)=b$ and also provided that for all $k$ strictly between $i$ and $j$ we have $f(k) \neq b$. If a is a word over $A$, we say $f$ contains successive occurrences of a at the intervals $J$ and $r+J$, where $r>0$, provided that $f \mid J$ and $f \mid(r+J)$ are copies of a and provided that for every $s$ such that $0<s<r$ we know $f \mid(s+J)$ is not a copy of a.

DEFINITION. Let a be a word over A. We define a set of natural numbers 0 (a) thus: $r \epsilon O(a)$ if and only if there exists a configuration $f$ and an interval $J$ such that $f$ contains successive occurrences of a at $\mathrm{J}$ and $\mathrm{r}+\mathrm{J}$.

EXAMPLE 8. Let $A=\{0,1\}$ and $a=10101$. We can have a configuration in which we have "overlapping" occurrences of a like this,

10101

10101 .

so that we must have $2 \epsilon O(a)$. If we try to set up successive "overlapping" occurrences thus, 
we see we have introduced a third occurrence of a in this fashion,

$$
\begin{gathered}
10101 \\
10101 \\
10101
\end{gathered}
$$

so that $4 \& 0(a)$. On the other hand, if we write $10101-\ldots-10101$, then we see that by filling in the blanks with 1 's we introduce no third occurrence of a. Therefore $6,7,8, \ldots \in O(a)$. As a matter of fact $5 \epsilon O(a)$ since 1010110101 contains exactly two occurrences of $a$. Hence $0(a)=\{2\} \cup[5, \infty)$.

LEMMA 2. Let $\mathrm{A}$ be a set containing 0 and 1 , and let a be a given, nonempty word over $A$. If there exists a natural number $m$ such that $a 0^{m}$ a contains a third occurrence of $a$, then for every natural number, the word $a 1^{n} a$ contains no third occurrence of a. PROOF. Suppose there are natural numbers $m$ and $n$ such that $a 0^{m} a$ and $a l^{n} a$ each contain a third copy of a. We will produce a contradiction.

Note first that the third copy of a must contain symbols from $0^{\mathrm{m}}$ and $1^{\mathrm{n}}$ so that a cannot be a constant word.

There are only certain ways the third occurrences of a could lie in $\mathrm{a}^{\mathrm{m}} \mathrm{a}$ and $\mathrm{al} \mathrm{l}^{\mathrm{n}} \mathrm{a}$, and consideration of these ways gives rise to cases.

Case 1. Suppose $\mathrm{a}=\mathrm{b} 0^{\ell}$ where $\mathrm{b}$ is a nonempty proper right factor of $\mathrm{a}$ and $1 \leq \varepsilon \leq \mathrm{m}$. We have $a=c b$ for some word $c$. If $|b| \leq \ell$, then $a=c b=b 0^{\ell}$ implies $b$ is a constant word made up of $0^{\prime} s$ and hence so is $a$. This is impossible, so $|b|>\ell$. Again from $a=c b=b 0^{\prime}$ it follows that $b=b_{1} 0^{2}$ where $b_{1}$ is a proper right factor of $b$. Then we must have $a=c b_{1} 0^{\prime}=b_{1} 0^{2 l}$. Note also that $\left|b_{1}\right|<|b|$. As we argued above, we may show that $\left|h_{1}\right|>l$ and hence that $a=c b_{2} 0^{2 l}=b_{2} 0^{3 l}$ where $b_{2}$ is a proper $r i g h t$ factor of $b_{1}$ and $' h_{2}^{\prime}=$ Clearly we can construct an infinite sequence $b_{1}, b_{2}, b_{3}, \ldots$ such that $b_{n+1}$ is a proper right factor of $b_{n}$ and $\left|b_{n}\right|>\left|b_{n+1}\right|>\theta$ for every $n$. Contradiction.

We also see from this proof that we cannot have $a=b l^{l}$ where $b$ is right factor of $a$ and $1 \leq \ell \leq n$, nor can we have $a=0^{\ell} b$ or $a=1^{l} b$ where $b$ is a left factor of $a$ and $1 \leq \ell \leq \mathrm{m}$ or $1 \leq \ell \leq \mathrm{n}$ respectively.

Case 2. We must be able to write $a=b_{0} 0{ }^{m} c_{0}=b_{1} l^{n} c_{1}$ where $b_{0}$ and $b_{1}$ are nonempty proper right factors of a and $c_{0}$ and $c_{1}$ are nonempty proper left factors of a. We may, without loss of generality, suppose that $\left|b_{0}\right| \leq\left|b_{1}\right|$.

We first show that for some natural number $k$ we have $a=\left(b_{0} 0^{m}\right)^{k}$ where $c$ is $a$ left factor of $b_{0} 0^{m}$. We know $a=c_{0} d$ for some word $d$. Suppose we can construct a sequence of words $c^{\prime}{ }_{0}, c^{\prime}{ }_{1}, \ldots, c^{\prime}{ }_{j}$ such that $c^{\prime}{ }_{i+1}$ is a proper right factor of $c^{\prime}{ }_{i}$ for $i=0,1, \ldots, j-1$ and $a=\left(b_{0} 0^{m}\right)^{j+1} c^{\prime}{ }_{j}=\left(b_{0} 0^{m}\right)^{j} c^{\prime}{ }_{j} d$. (Certainly this is true for $j=0$ with $\left.c^{\prime}{ }_{0}=c_{0}\right)$ Suppose $\left|c^{\prime}{ }_{j}\right| \leq\left|b_{0} 0^{m}\right|$. From $\left(b_{0} 0^{\mathrm{m}}\right)^{j+1} c^{\prime}{ }_{j}=\left(b_{0} 0^{m}\right)^{j} c^{\prime}{ }_{j} d$ we see that $c^{\prime}{ }_{j}$ must be a left factor of $b_{0} 0^{m}$. If we set $c=c^{\prime}{ }_{j}$, we see from $a=\left(b_{0} 0^{m}\right)^{j+1} c^{\prime} j$ that we are done. Suppose on the other hand that $\left|c^{\prime}{ }_{j}\right|>\left|b_{0} 0^{m}\right|$. From $\left(b_{0} 0^{m}\right)^{j+1} c^{\prime}{ }_{j}=\left(b_{0} 0^{m}\right)^{j} c^{\prime}{ }_{j} d$ we see that $c^{\prime}{ }_{j}=b_{0} 0^{m} c^{\prime}{ }_{j+1}$, which makes $c^{\prime}{ }_{j+1}$ a proper right factor of $c^{\prime}{ }_{j}$, and we must have $a=\left(b_{0} 0^{m}\right)^{j+2} c^{\prime}{ }_{j+1}=\left(b_{0} 0^{m}\right)^{j+1} c^{\prime}{ }_{j+1} d$. As the lengths of the $c_{i}$ 's are decreasing, we must ultimately be able to find one which we can use as c. We know from the fact that $a=\left(b_{0} 0^{m}\right)^{k} c=b_{1} 1^{n} c_{1}$, where $c$ is a left factor of $b_{0} 0^{m}$, that we can write $b_{0}$ in the form $b_{0}=e^{n} f$. Since $\left|b_{0}\right| \leq\left|b_{1}\right|$ and $\left(b_{0} 0^{m}\right)^{k} c_{c}=b_{1} l^{n_{c}} c_{1}$, 
it follows there must be a $j$ such that $b_{1}=\left(b_{0} 0^{m}\right)^{j} e=\left(b_{0} 0^{m}\right)^{j-1} b_{0} 0^{m} e=\left(b_{0} 0^{m}\right)^{j-1}$ el ${ }^{n} 0^{m} e$. It follows from this and the fact that $b_{1}$ is a right factor of a that fome must be a right factor of a. Recall that $b_{0}=e 1^{n} f$ is also a right factor of a. Thus one of the two words $f 0^{\mathrm{m}} \mathrm{e}$ and $\mathrm{el}{ }^{\mathrm{n}} \mathrm{f}$ must be a right factor of the other. Suppose $\mathrm{f} 0^{\mathrm{m}} \mathrm{e}$ is a right factor of $e 1^{n} f$ and for every word $x$ let $Z(x)=$ the number of 0 's in $x$. Then $Z\left(f 0^{m} e\right)=Z(f)+m+Z(e) \leq Z\left(e 1^{n} f\right)=Z(e)+Z(f)$. This is impossible. A similar argument based on counting the number of $l^{\prime}$ 's in a word disposes of the possibility that el ${ }^{n} f$ is a right factor of $\mathrm{fo}^{\mathrm{m}} \mathrm{e}$.

This lemma gives us information about $0(a)$; it tells us that as long as $A$ has at least two elements, at least one of those elements, say 1 , can be inserted between two copies of a as often as we wish without inadvertently producing a third copy of a, and hence $|a|+n \in 0(a)$ for $n=1,2,3, \ldots$ This in turn says that when computing 0 (a), we need check only $1,2, \ldots,|a|$.

PROPOSITTON 7. If A contains at least two elements and a is a given, nonempty word cver $A$, then the interval $[|a|+1,(x)$ in $Z$ is contained in $0(a)$.

This result gives significance to the following characterization of $\operatorname{ran}(\mathrm{T})$ and $G(\mathrm{~T})$ when $\mathrm{T}$ is a characteristic parallel map:

PROPOSITJON 8. Let $T$ be a characteristic map for $\left(a_{1}, a_{2}, \ldots, a_{n}\right)$. We identify $\left(a_{1}, a_{2}, \ldots a_{n}\right)$ with the word $a=a_{1} a_{2} \ldots a_{n}$. Then $f \in \operatorname{ran}(T)$ if and only if

(1) $f$ takes on only the values 0 and 1 , and

(2) if $i$ and $j$ mark successive occurrences of $l$ in $f$ with $i<j$, then $j-i \in O(a)$.

lien (,T) is of course the set of configurations which fail to satisfv (1) or (2).

PROOF. It is trival that members of $\operatorname{ran}(T)$ satisfy (1) and (2). Suppose f sat lifit: (1) and (2) and $\mathrm{W}$ is the window for $T$. We begin construction of a configuration $g$ by placing a copy of a on every interval $k+W$ for which $f(k)=1$. If two such intervals $k_{1}+W$ and $k_{2}+W$ happen to overlap, we know from the definition of $0(a)$ that we shall be able to contruct both copies simultaneously. If there are intervals between the copies of a where $g$ has been assigned no value, we know from Lemma 2 a constant value can be assigned there which will not produce any extra copies of a word a. In this way we can construct $g$ in such a way that $g$ has a copy of a at $k+W$ if and only if $f(k)=1$, and thus $\mathrm{Tg}=\mathrm{f}$.

PROPOSITION 9. Suppose $A$ has at least three elements and $T$ is a characteristic map for the word a over A. Then $f \in W G(T)-G(T)$ if and only if

(1) $\mathrm{Tf}=\mathrm{S}^{\ell} \mathrm{f}$ for some integer $\ell$,

(2) $f$ is not the constant 0 configuration,

(3) $f$ has neither a first nor a last integer at which it takes on the value 1 , and

(4) if $i$ and $j$ mark successive occurrences of $l$ in $f$, then $|i-j| \leq|a|$.

PROOF. Let $W=$ the window for $T$ and assume $0,1,2 \in \mathrm{A}$.

Suppose $f \in W G(T)-G(T)$. Proposition 1 implies (1). By Lemma 2 we may, without loss of generality, assume the word al a contains no third occurrence of a for every natural number $n$. This implies the constant configuration $g$ of value 1 contains no copies of $\mathrm{a}$, and hence $\mathrm{Tg}=0$ (the constant configuration of value 0 ). Now if we examine the proof of Lemma 2, we find the only property of 0 and 1 used there was the fact that they are distinct; we could just as easily have used 0 and 2 . We deduce from this 
that there can be at most one element of $A$, say 0 , such that a word of the form a $0^{m} \cdot 1$. where $m$ is a natural number, can have a third occurrence of a. So we can, without loss of generality, also assume that for every natural number $n$ the word a $2^{n}$ a contains no third occurrence of a. Thus the constant configuration of value $2, h$, satisfies $T h=0$. Since the constant zero configuration has two pre-images, it cannot be a weak Garden of Eden, so (2) holds. For the rest of this first half of the proof we continue to assume every $a 1^{n} a$ and $a 2^{n} a$ contains no third occurrence of $a$. Suppose $j$ is the last integer at which $f(j)=1$. Let $g=s^{-l} f$ where $T f=s^{2} f$; this means $T g=f$. We must have a copy of a at $j+W$ in $g$. Suppose $r$ is the largest integer in $j+w$. Define

$$
\begin{aligned}
& g_{1}(i)= \begin{cases}g(i) & \text { if } i \leq r \\
1 & \text { if } r+1 \leq i\end{cases} \\
& g_{2}(i)= \begin{cases}g(i) & \text { if } i \leq r \\
2 & \text { if } r+1 \leq i .\end{cases}
\end{aligned}
$$

Then $g_{1}$ and $g_{2}$ have copies of $a$ at $k+W$ if and only if $g$ does, so $T g_{1}=T g_{2}=T g=f$. So $f$ must have distinct pre-images, an impossibility, and we conclude there is no last integer at which $\mathrm{f}$ takes on the value 1 . Similarly there can be no such first integer. Heme (3) holds. Iet us continue with $g$ as defined above and suppose integers $i$ and malk successive occurrences of 1 in $f$ where $j>j$ and $j-i>|a|$. This means $g$ his copies of a at $i+W$ and $j+W$ and also that between these two intervals there is an interval $J$ which overlaps no copy of $a$. By changing the values of $g$ on $J$ first to 1 and then to 2, we can construct two different pre-images for $f$. Since this is impossible, (4) holds.

Now suppose $f$ is a configuration satisfying (l) - (4). We know from (1) that $\mathrm{s}^{-l_{\mathrm{f}}}$ is a pre-image of $f$ under $T$. Suppose $T g=f$. For every $j$ such that $f(j)=1$, we must have a copy of a at $j+W$ in $g$. Taking this fact in conjunction with (2) - (4), we see that $g$ is covered by overlapping copies of a and hence is uniquely determined; thus $g=S^{-\ell} f$. So $f \in W G(T)-G(T)$.

ACKNOWLEDGEMENT. This work was partially supported by a grant from the Division of Sponsored Research, The University of Central Florida, in the summer of 1983.

\section{REFERENCES}

1. AMOROSO, S. and COOPER, G. The Garden-of-Eden Theorem for Finite Configurations, Proc. Amer. Math. Soc. 26, 158-164 (1970).

2. AMOROSO, S., COOPER G. and PATT, Y. Some Clarifications of the Concept of a Gardenof-Eden Configuration, J. Comput. System Sci. 10, 77-82 (1975).

3. MOORE, E.F. Machine Models of Self-reproduction, in Essays on Cellular Automata (A.W. Burks, Ed.) 187-203, Univ. of Illinois Press, Urbana, Illinois, 1970.

4. MYHILL, J. The Converse of Moore's Garden-of-Eden Theorem, in Essays on Cellular Automata (A.W. Burks, Ed.), 204-205, University of Illinois Press, Urbana, Illinois, 1970.

5. SMITH, A.R. Introduction to and Survey of Polyautomata Theory, in Automata, Languages, Development (A Lindenmayer, G. Rozenberg, Ed.) 405-424, North-Holland, Amsterdam, 1976.

6. SKYUM, S. Confusion in the Garden of Eden, Proc. Amer. Math. Soc., 50, 332-336 (1975).

7. HEDLUND, G.A. Endomorphisms and Automorphisms of the Shift Dynamical System, Math. Systems Theory $\underline{3}, 320-375$ (1969). 
8. NASU, M. I ocal Maps Inducing surjective Global Maps of One-dimensional Tessellation Automata, Math Systems Theory 11, 327-351 (1978).

9. NASU, M. An Interconnection of Local Maps Inducing onto Global Maps, Discrete Applied Math. 2, 125-150 (1980).

10. RICHARDSON, D. Tessellations with Local Transformations, J. of Computer and Sys. Sci. 6, 373-388 (1972).

11. AMOROSO S. and PATT, Y. Decision Procedure for Surjectivity and Injectivity of Parallel Maps for Tessellation Structures, J. Comput. System Sc1. 6, 448-464 (1972). 


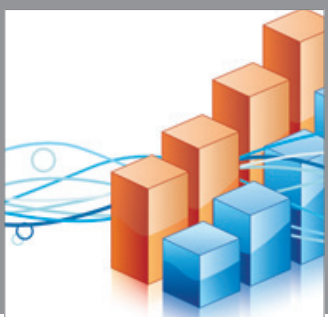

Advances in

Operations Research

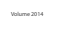

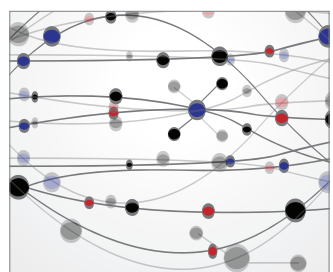

\section{The Scientific} World Journal
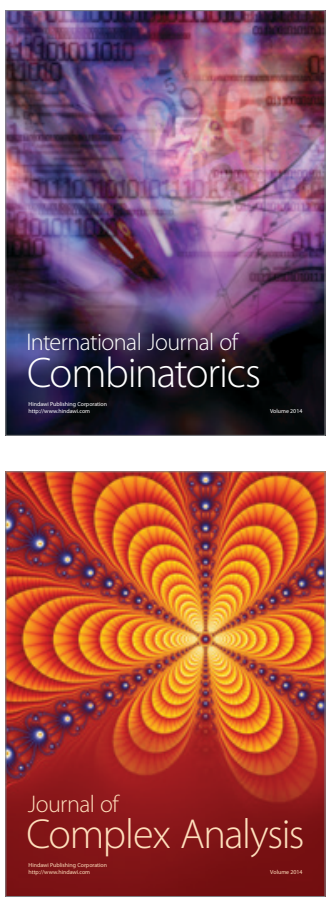

International Journal of

Mathematics and

Mathematical

Sciences
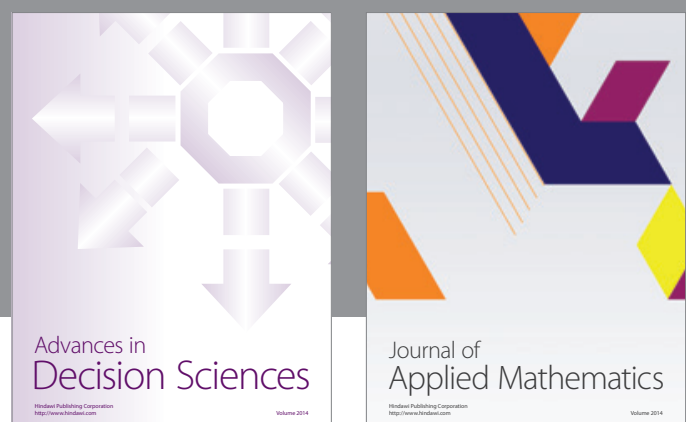

Journal of

Applied Mathematics
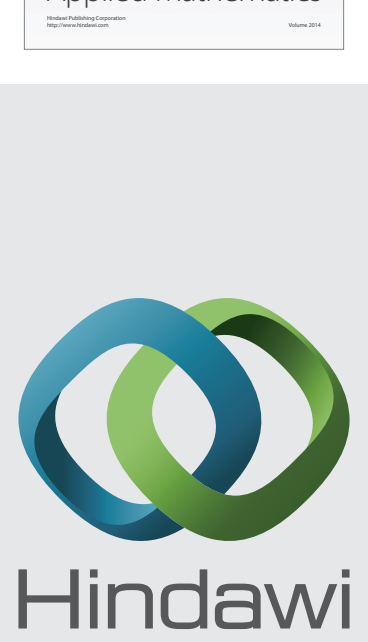

Submit your manuscripts at http://www.hindawi.com
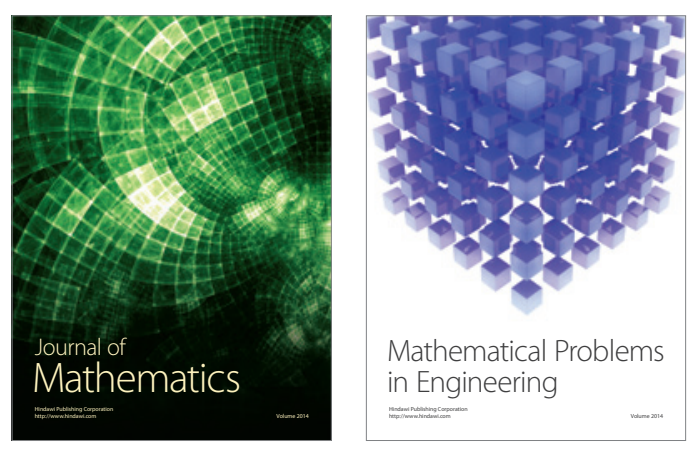

Mathematical Problems in Engineering
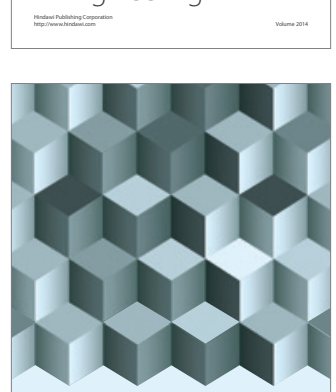

Journal of

Function Spaces
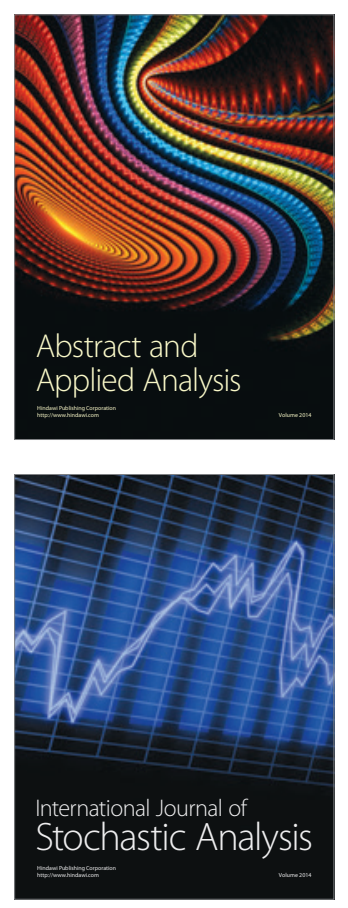

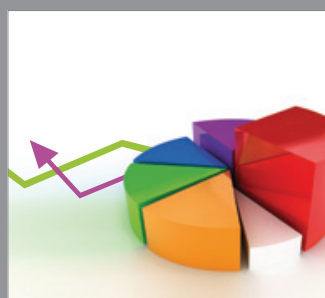

ournal of

Probability and Statistics

Promensencen
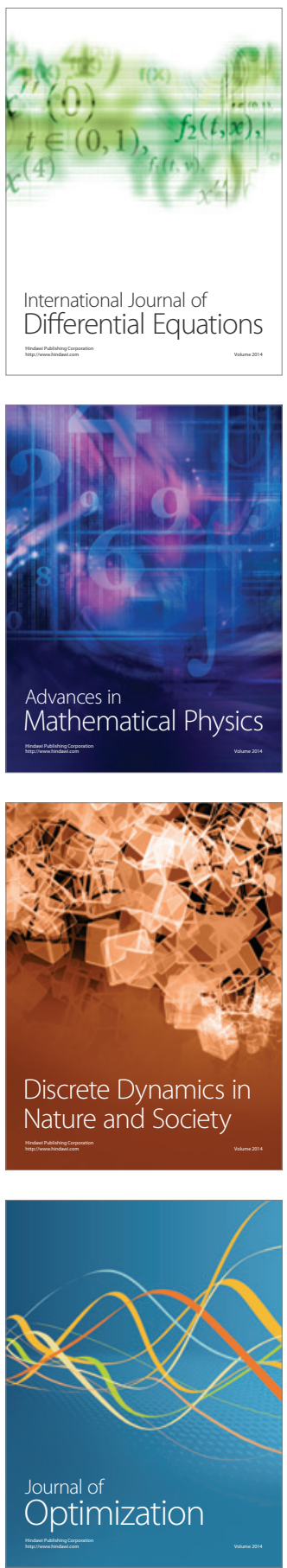